\title{
La Docencia en Ciencias Medioambientales en la Universidad Autónoma de Madrid. Teoría y Práctica
}

\author{
Esperanza Martín ${ }^{(1)}$, Oscar Fernández ${ }^{(2)}$ y Jacobo Atienza(3) \\ (1) Dpto. de Química Física Aplicada. Módulo 14. Facultad de Ciencias. UAM. Carretera de Cantoblanco \\ s/n. 28049. Madrid. España (e-mail: esperanza.martin@uam.es) \\ (2) C/ Vázquez de Mella, 3 - 1ํdcha. Oviedo. 33012. Asturias. España (e-mail: oscarfmarcos@gmail.com) \\ (3) C/ Caleruega, 61 - 2ํㅡ. 28033. Madrid. España (e-mail: jacobo@atienzaramos.com)
}

Recibido May. 5, 2015; Aceptado Jun. 3, 2015; Versión final Ago. 1, 2015, Publicado Dic. 2015

\section{Resumen}

Se presenta un estudio sobre la importancia de habituar a los alumnos de Ciencias Medioambientales a contrastar las teorías sobre generación, reactividad y evolución de contaminantes atmosféricos, con medidas experimentales, o con buenas bases de datos disponibles en Internet. Se aplicó el concepto al análisis de datos recogidos por los autores en los analizadores automáticos del campus de la Universidad Autónoma de Madrid sobre óxidos de nitrógeno, ozono, dióxido de azufre, partículas en suspensión, e intensidad de emisión solar. Las medidas se realizaron en tres periodos anuales y fueron almacenadas en el sistema para adquisición de datos conectado a los analizadores. Los resultados presentados resumen la concentración de los contaminantes en las muestras de aire analizadas, mostrando el bajo nivel de contaminación del campus. Los resultados también ratifican los conceptos teóricos sobre origen y evolución de estos contaminantes en la atmósfera para el lugar y los tiempos seleccionados.

Palabras clave: análisis de contaminantes, contaminantes atmosféricos, datos ambientales, enseñanza en ciencias ambientales

\section{The Teaching of Environmental Sciences at the Autonomous University of Madrid. Theory and Practice}

\begin{abstract}
This study shows the importance of habituating Environmental Sciences students to comparing the theories about generation, reactivity and evolution of atmospheric pollutants with experimental measurements or with good databases available in the Internet. This concept was applied to the analysis of the data collected in the automatic analyzers located in the campus of the Autonomous University of Madrid regarding nitrogen oxides, ozone, sulfur dioxide, suspended particles and solar emission intensity. These measures were carried out in three periods and were recorded in a data logger connected to the analyzers. The results presented summarize the concentration of those pollutants in the air samples analyzed, showing a low pollution level at the campus. Results also ratify the theoretical concepts about the origin and evolution of those pollutants in the atmosphere for the selected place and time.
\end{abstract}

Keywords: pollutants analysis, atmospheric pollutants, environmental data, environmental sciences teaching 


\section{INTRODUCCIÓN}

El trabajo en docencia y dirección de proyectos en la Licenciatura de Ciencias Medioambientales de la Universidad Autónoma de Madrid (UAM) desde la perspectiva de la Química (Atkins, 2012), nos llevó a plantear cómo generar una mayor interacción entre la enseñanza teórica de la asignatura Contaminación Atmosférica (Finlayson-Pitts, 2000; Spiro, 2003) y las prácticas sobre medidas directas de los contaminantes atmosféricos en aire. Desde un punto de vista científico y situándonos en la asignatura citada, antes de introducir sofisticadas teorías sobre modelos de cambio climático, se debe trasmitir a los alumnos la importancia de contrastar con la medición experimental, siempre que sea posible, las teorías y datos transferidos como válidos en las clases teóricas. En este trabajo se trata de conectar al alumno con los fundamentos técnico-científicos para la medida de las concentraciones de los contaminantes gaseosos más habituales en entornos urbanos en un primer paso y para ser capaces de relacionarlos en un segundo paso con aspectos del entorno en que se realizan las medidas: situación geográfica, fuentes de contaminación que los producen, hora del día y época del año en que fueron tomadas.

La contaminación ambiental es uno de los paradigmas a los que se enfrenta la sociedad en el siglo XXI. Se sabe que de la calidad del aire puede depender la salud de plantas, animales y personas. El modo de vida establecido en las grandes urbes, integradas por millones de personas en ámbitos reducidos, que necesitan espacios habitables y medios de transporte, genera una contaminación ambiental específica, denominada "smog fotoquímico" (Baird, 2001) que acarrea problemas sobre el sistema respiratorio y la salud de las personas que las habitan. Publicaciones recientes en periódicos (ABC, 2015), o en revistas científicas sobre ciudades como Pekin (Lin-Yu, 2014), algunas del este de Indonesia (Shibata, 2014) 0 sobre tres ciudades norteamericanas (Gass, 2015), muestran los efectos de las concentraciones de partículas en suspensión, ozono y óxidos de nitrógeno sobre la salud de adultos y niños. Los principales contaminantes en las grandes urbes son: los restos de hidrocarburos y sus radicales, el dióxido de azufre y los tres citados en el punto anterior. Las partículas en suspensión con diámetro inferior a 10 $\mu \mathrm{m}$, denominadas $\mathrm{PM}_{10}$, son las que se han medido habitualmente, si bien en los últimos años se ha extendido el estudio sobre su toxicidad y su control a las de menor tamaño, $P_{2,5 .}$. (Steinle, 2015; Bin Luo, 2014). La medida de la concentración de estos contaminantes en aire, es por ello una práctica habitual en las ciudades españolas y europeas con más de 200.000 habitantes. Estas medidas se realizan en los denominados analizadores automáticos homologados. Las que se tomaron para este trabajo fueron hechas en los analizadores del aula medioambiental, situada en el campus de la UAM, a unos $15 \mathrm{Km}$ de distancia en dirección norte del centro geográfico de la ciudad de Madrid y en las coordenadas, Latitud $40^{\circ} 32^{\prime}$ y Longitud $3^{\circ} 41^{\prime}$.

La importancia dada a la influencia del medio ambiente sobre la salud se pone de manifiesto por la existencia de publicaciones científicas de interés en décadas anteriores (Künzli, 2005; Ballester, 1999; Lave, 1970). La transversalidad y amplitud que acompaña el estudio de los problemas medioambientales abarcan otras áreas como la economía, (Servicio de Estudios de la Caixa, 2009), la sociología en ambientes urbanos densamente poblados, o su proyección en la legislación de un país o territorio (AENOR, 2006) y puede llevar a algunos docentes a perder la perspectiva de la importancia de, añadir a las clases teóricas de los alumnos universitarios, prácticas basadas en la medida experimental de la concentración de los contaminante del entorno en que viven. Se necesitan equipos automáticos precisos, homologados y revisados periódicamente, para la medida directa en tiempo real de los contaminantes atmosféricos, así como sistemas de acumulación de datos para su posterior análisis y evaluación (SIR 1, 2010). Como no siempre es posible disponer de medios técnicos, entre otras cuestiones por su coste económico y de mantenimiento, sugerimos también a los alumnos en prácticas, acudir a las páginas webs: locales (Web Ayuntamiento de Madrid), regionales (Web. Comunidad de Madrid) o nacionales (Web del Ministerio de Medio Ambiente.), y analizar las secuencias de datos almacenadas en régimen horario/diario o bien el mensual/anual, que refleja la evolución de la contaminación en diferentes épocas del año para la ciudad elegida, en este caso Madrid.

Esta información puede ayudar en la preparación del alumno sobre la contaminación atmosférica del entorno que habita. En el caso de que tampoco haya webs locales, existen algunas páginas internacionales, como la (Web de la Agencia Europea de Medioambiente) y la (Web de la Organización Meteorológica Mundial), que puedan dar información sobre concentración y evolución de contaminantes de fondo en la atmósfera en los últimos decenios. Las leyes españolas, basadas en la transposición de Directivas del Consejo de Europa (Agencia Europea del Medio Ambiente, 2001), sitúan los límites de la concentración de los contaminantes objeto de nuestro análisis, en el rango de concentraciones de ppb, ppm o $\mu \mathrm{g} / \mathrm{m}^{3}$ en aire (RD 102/Boletín Oficial, 2011).

En este trabajo se recopilan medidas de contaminantes urbanos, cuya toxicidad sobre seres vivos esta estudiada, y se aporta la representación gráfica de los valores de los contaminantes para: 
a) Óxidos de nitrógeno $\mathrm{NO} / \mathrm{NO}_{2}$ y ozono por un lado. Estos contaminantes peligrosos para el sistema respiratorio, participan de un ciclo fotolítico en el que su formación y destrucción está asistida por la radiación solar. Por ello se aportan datos sobre intensidad de la radiación solar en algunas de las gráficas (Künzli, 2012; Figueruelo, 2004; World Health Organization, 1992). Reacciones del ciclo:

$$
\begin{aligned}
& \mathrm{NO}_{2}+\mathrm{hv} \rightarrow \mathrm{NO}+\mathrm{O} \quad \lambda \leq 380 \mathrm{~nm} \\
& \mathrm{O}+\mathrm{O}_{2} \rightarrow \mathrm{O}_{3} \\
& \mathrm{O}_{3}+\mathrm{NO} \rightarrow \mathrm{O}_{2}+\mathrm{NO}_{2}
\end{aligned}
$$

b) Dióxido de azufre $\mathrm{SO}_{2}$ y partículas en suspensión $\mathrm{PM}_{10}$ por otro lado, ya que está comprobado mediante estudios médicos epidemiológicos, cómo la combinación de ambas especies genera problemas o agrava los existentes sobre el sistema respiratorio (Tasic, 2014; Vineis, 2006). Si bien nosotros solo presentamos datos sobre partículas en suspensión de diámetro promedio inferior a 10 $\mu m\left(\mathrm{PM}_{10}\right)$, la tendencia actual, como ya se indicó, es medir las de diámetro inferior a $2.5 \mu \mathrm{m}\left(\mathrm{PM}_{2.5}\right)$. Su pequeño tamaño puede llevarlas hasta los alveolos pulmonares y presentan mayor dificultad para su eliminación que las PM10. Se trata de trasmitir la idea de que los alumnos sean proactivos en la toma directa de datos sobre contaminantes en aire, el análisis de las causas u origen de ellos, los mecanismos físico-químicos y especies químicas que contribuyen a su crecimiento o dispersión y por último las posibles soluciones para disminuir su presencia o para su eliminación.

\section{MÉTODOS DE MEDIDA DE LOS CONTAMINANTES ATMOSFÉRICOS ANALIZADOS.}

\section{a) Ciclo de los óxidos de nitrógeno}

Las medidas de los óxidos de nitrógeno se realizan en un analizador automático (SIR SA. 2, 2001), calibrado para las medidas en determinados intervalos de presión y temperatura. La cuantificación de estos compuestos está basada en la luminiscencia producida en la siguiente reacción:

$\mathrm{O}_{3}+\mathrm{NO} \rightarrow \mathrm{O}_{2}+\mathrm{NO}_{2}^{*}$

En ella el dióxido de nitrógeno posee una energía de excitación, que al emitirla en forma de fotones en un tiempo de ns, puede dar una relación cuantitativa de las moléculas de NO presentes en la muestra de aire: $\mathrm{NO}_{2}{ }^{*} \rightarrow \mathrm{NO}_{2}+$ hv. La estequiometria 1:1 de la reacción, la conveniente amplificación de la emisión con un fotomultiplicador y los componentes electrónicos, nos dan la concentración en partes por billón (ppb) del NO en intervalos de tiempo de 3 minutos.

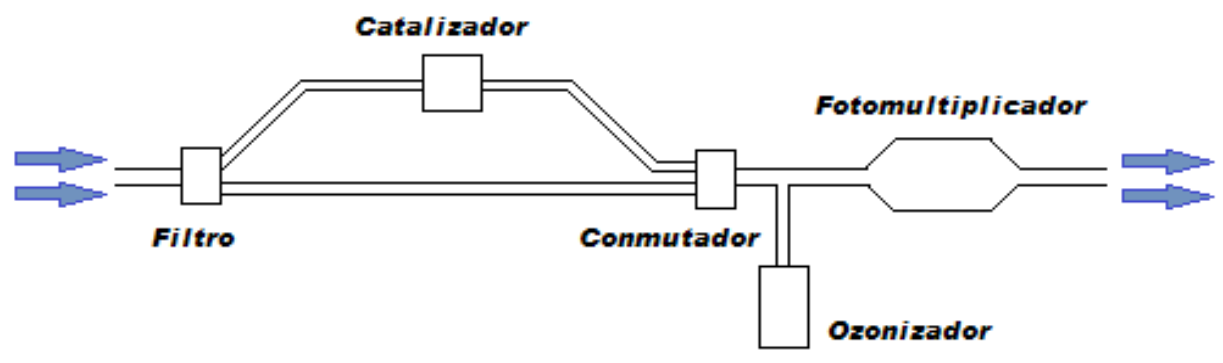

Fig. 1: Esquema de funcionamiento del analizador automático de los NOx (Fernández, 2010).

Para obtener la concentración del $\mathrm{NO}_{2}$, el sistema tiene un conmutador que hace que el aire pase por un catalizador que lo reduce a NO y lo mide por el mecanismo anterior mencionado. Ver esquema en la Fig.1. Se debe recordar que la fuente principal de emisión del contaminante primario que es el NO, procede de la combustión de hidrocarburos producida en los vehículos de transporte, industrias y calefacciones. A la T ambiente de $298 \mathrm{~K}$, la constante de equilibrio de la reacción de nitrógeno y oxígeno molecular $\mathrm{k}_{\mathrm{p}}=1.10^{-30}$; es tan baja, que hace improbable la reacción de los principales componentes del aire: $\mathrm{N}_{2}+\mathrm{O}_{2} \leftrightarrow 2 \mathrm{NO}_{2}$; a $1000 \mathrm{~K}$ el valor $\mathrm{K}=7.9 .10^{-9}$ permite la reacción en mayor proporción.

El paso de $\mathrm{NO}$ a $\mathrm{NO}_{2}$, está controlado en la atmósfera por el ciclo que se muestra en la Fig.2 y está favorecido a T ambiente (Figueruelo, 2004). De este gráfico se puede deducir que la radiación solar favorece la disociación del $\mathrm{NO}_{2}$, la formación del $\mathrm{O}_{3}$ y la posterior reacción de esta molécula con el $\mathrm{NO}$ liberado en las combustiones de los motores, generando nuevamente $\mathrm{NO}_{2}$. En la concentración medida para estos contaminantes en el campus de la UAM influyen factores tales como: número de vehículos 
privados o públicos de acceso, hora del día en que se toman las muestras y época del año ya que ambas determinan la intensidad de la radiación solar y la temperatura media del aire. Por ello se tomaron muestras durante las $24 \mathrm{~h}$, los siete días de la semana y en los meses de noviembre, marzo y mayo. El entorno geográfico también puede influir en la renovación de las masas de aire y de sus contaminantes.

Las medidas de ozono se realizaron en un analizador automático (SIR 3, 2001), basado en la absorción de fotones de una lámpara de luz UV (intensidad I) por la molécula, y la comparación con los fotones de intensidad $I_{0}$ en una muestra de aire limpia de ozono y otros materiales absorbentes a esa longitud de onda; a bajas concentraciones como en las que aparece el $\mathrm{O}_{3}$ en la atmósfera (ppm), la absorbancia sigue la Ley de Lambert-Beer (Born, 2002). Todo los datos recogidos en el sistema acumulador de datos para estos contaminantes fueron tratados y representados mediante el programa informático IBM SPSS (Statistical Package for the Social Sciencies) (Fernández, 2010).

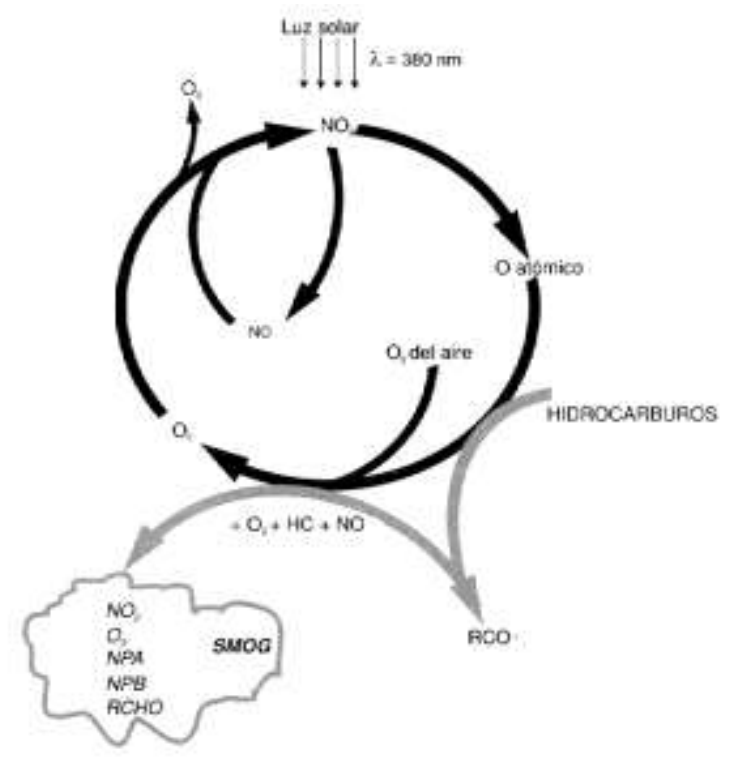

Fig. 2: Ciclo fotolítico de los $\mathrm{NO}_{\mathrm{x}}$, alterado por la presencia de hidrocarburos.

b) Medida de dióxido de azufre y de partículas $P M_{10}$

La medida de la concentración de $\mathrm{SO}_{2}$ se realizó en un analizador automático basado en la espectroscopia de fluorescencia (SIR 4, 2001). Esta molécula tiene la propiedad de emitir fotones en un intervalo temporal de ns, al ser previamente iluminada con una lámpara de zinc de luz UV.

$\mathrm{SO}_{2}+\mathrm{hv} \rightarrow \mathrm{SO}_{2}{ }^{*} \rightarrow \mathrm{SO}_{2}+\mathrm{hv}^{\prime}$ (fluorescencia)

La intensidad de fotones es proporcional al número de moléculas de dióxido de azufre en aire. Su concentración (ppb), ha disminuido en las ciudades españolas en los 10-15 últimos años, debido al escaso uso del carbón en calefacciones y al uso de gasolinas y gasóleos depurados de compuestos con azufre.

La medida de la concentración de partículas $\mathrm{PM}_{10}$ se realizó mediante un captador de partículas de absorción Beta automático, modelo FH62I-N, realizada a intervalos de 30 segundos. La radiación inicial Beta $\mathrm{N}_{0}$, queda atenuada por el efecto que ejercen las partículas de la muestra de aire, al quedar fijadas sobre una cinta transparente, que las retiene y se mueve a velocidad controlada.

$\mathrm{N}=\mathrm{N}_{0} \cdot \mathrm{e}^{-k \mu}$

Donde $\mathrm{N}_{0}$ es el número de impulsos registrados en el filtro limpio; $\mathrm{N}$ es el número de impulsos cuando hay partículas, $\mathrm{K}$ es la constante física de medida y $\mu$ es el coeficiente de atenuación lineal. Los datos recogidos fueron representados mediante el programa informático IBM SPSS (Atienza, 2010).

\section{RESULTADOS GRÁFICOS DE LAS MEDIDAS REALIZADAS}

Como se indicó en la introducción, el trabajo se diseñó para realizar medidas durante las 24 horas en siete días consecutivos de los meses de marzo, mayo y noviembre. En la Fig.3 se observan para el mes de marzo. Los valores de las concentraciones de los contaminantes del ciclo fotolítico a lo largo de un día de entre-semana de marzo en $\mu \mathrm{g} / \mathrm{m}^{3}(1,2,3)$ aparecen muy rebajadas al superponerlas con la intensa emisión de la radiación solar en $\mathrm{W}$ (vatios) $/ \mathrm{m}^{2}(4)$, ambas en ordenadas. Con ello se quiere mostrar que los máximos del $\mathrm{O}_{3}$ coinciden con las horas de mayor intensidad de radiación, al contrario de los máximos de los $\mathrm{NO}_{x}$. 


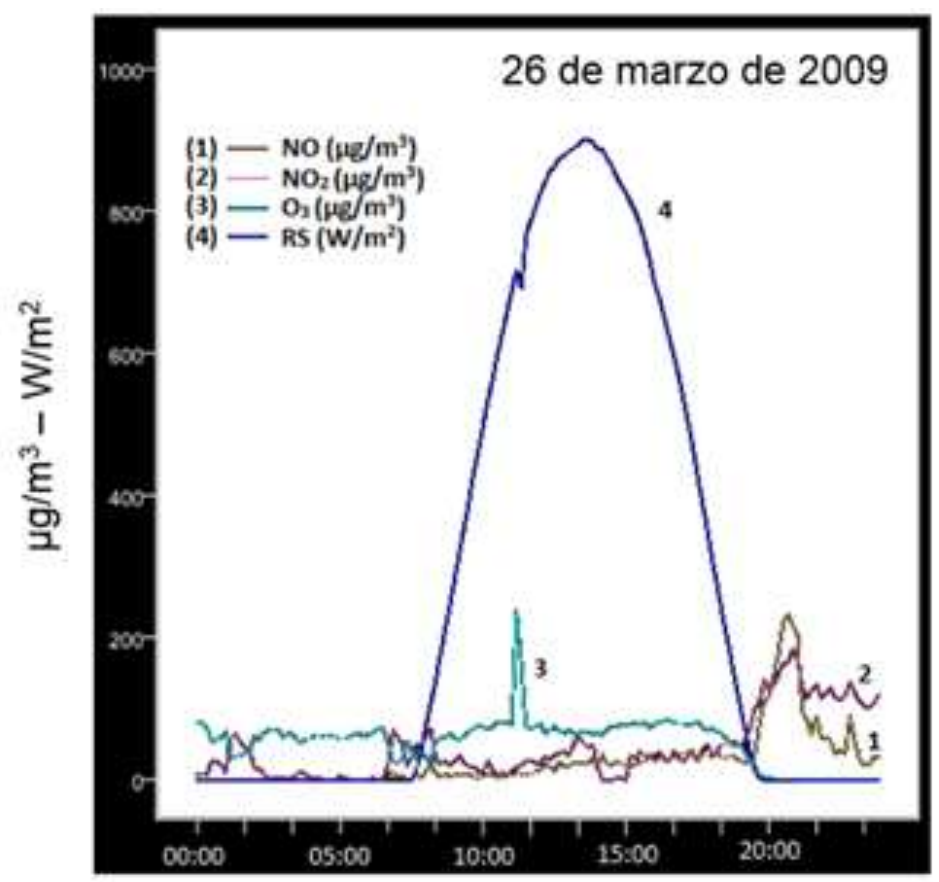

Fig. 3: Variación horaria de los contaminantes indicados y de la radiación solar

En la Fig.4 se visualiza con mayor claridad que la concentración de ozono (3) aumenta en las horas centrales del día, cuando la radiación solar es más intensa y disminuye a partir de las 18.0h. En ese momento aumenta extraordinariamente la concentración de los óxidos de nitrógeno, especialmente la del monóxido (1), coincidiendo con la vuelta a casa de los vehículos y con el hecho de que la radiación solar ya no disocia al $\mathrm{NO}_{2}(2)$, de acuerdo con el ciclo de la Fig.2.

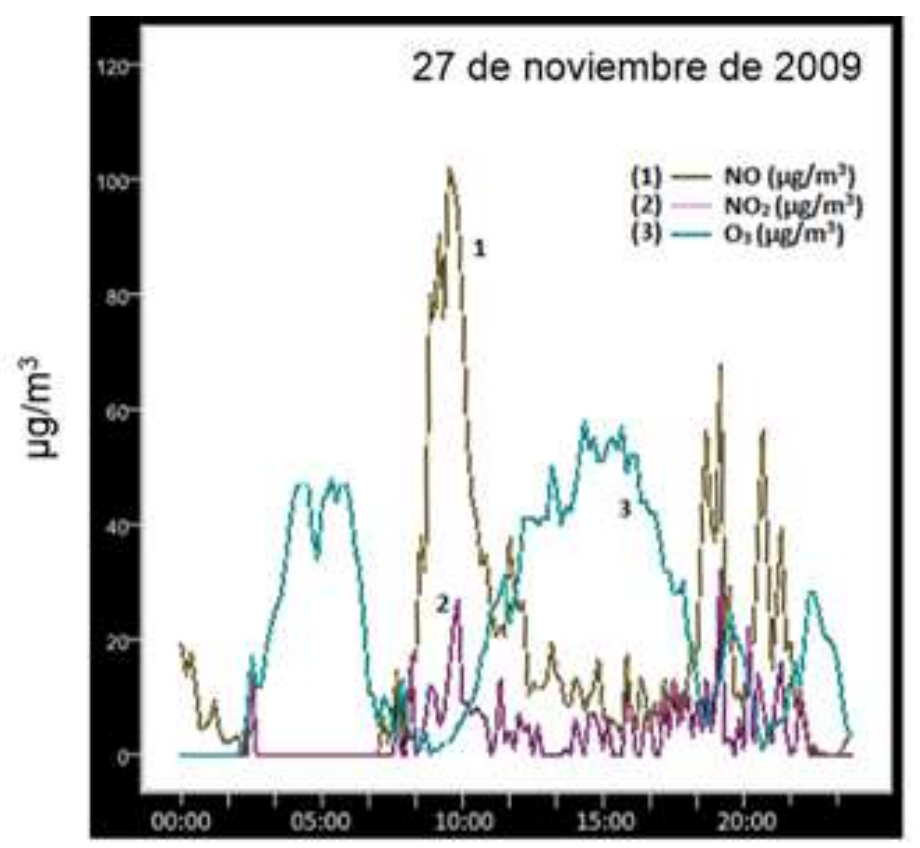

Fig. 4: Variación diaria/horaria de los contaminantes del ciclo fotolítico

El otro pico de ambos óxidos se produce entre las 7.0 y las 9.0h, correspondiendo con el inicio de la jornada laboral y con el número máximo de vehículos en el campus. La existencia de ozono en horas nocturnas está asociada a reacciones secundarias de restos de la combustión incompleta de los hidrocarburos y sus radicales. La Fig.5 muestra los picos de entrada y salida de vehículos en el campus. Se pretende comprobar experimentalmente que los contaminantes proceden de fuentes móviles, con un gráfico de las entradas y salidas de vehículos en el campus un día intermedio de la semana. Los datos proceden de una Memoria de Licenciatura (Romero, 2005). 


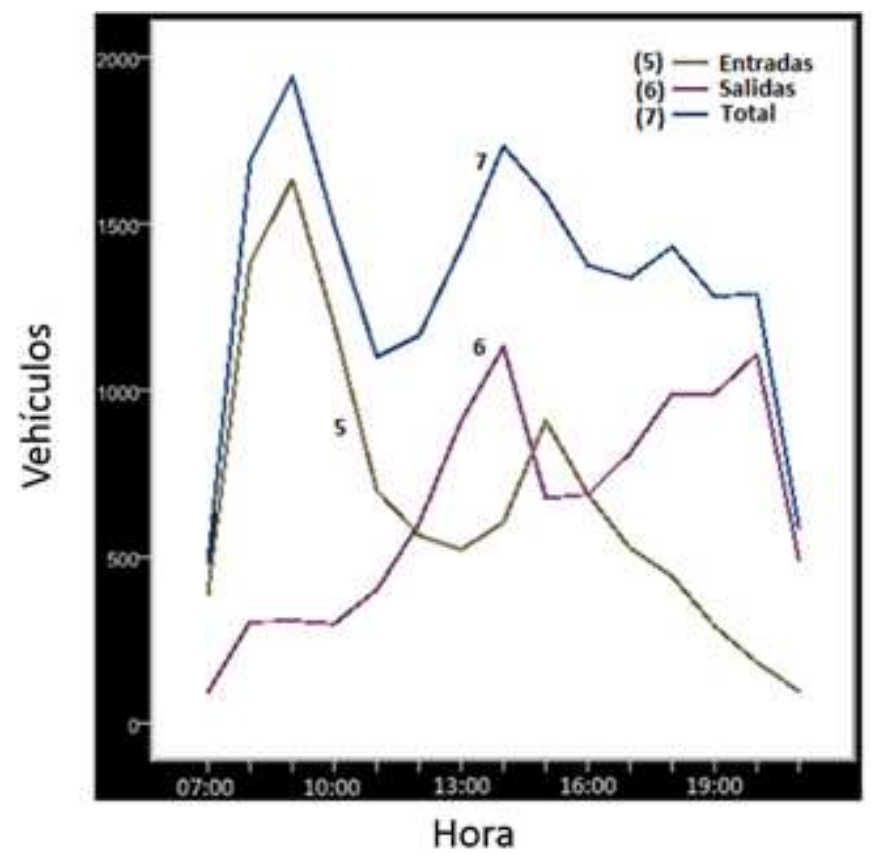

Fig. 5: Gráfica horaria de entradas y salidas de vehículos en el campus de la UAM

Se observa que entre 8.30-9.0h de la mañana entra el mayor número de vehículos, mientras que la salida está más escalonada con picos claros a las 14.0 y a las $20.0 \mathrm{~h}$, cuando finalizan turnos de clase de mañana y de tarde. Se puede observar la correlación entre vehículos en el campus, Fig.5 y concentraciones máximas de $\mathrm{NO}_{x}$, Fig.4 y Fig.3, además de la influencia de la radiación solar en el ciclo de los contaminantes considerados. En el caso que se analiza, las fuentes fijas han de tener escasa influencia sobre la concentración de contaminantes, excepto los generados por el encendido de las calefacciones en otoño - invierno. El campus está situado lejos de centrales térmicas, de industrias contaminantes y de núcleos urbanos grandes. Se considera que el calor debido al efecto invernadero, generado por la concentración de fondo de moléculas de $\mathrm{CO}_{2}$ en la atmósfera, actúa como factor constante sobre la temperatura media del aire, para un día sin incidentes reseñables. Con este supuesto se considera que la temperatura del aire procede del ciclo diario de la radiación solar, que a su vez interviene en el ciclo de los contaminantes $\mathrm{NO}_{x}$ y $\mathrm{O}_{3}$ analizados. En la Fig.6 se muestra la correspondencia a lo largo de los datos horarios recogidos en día de entre-semana.

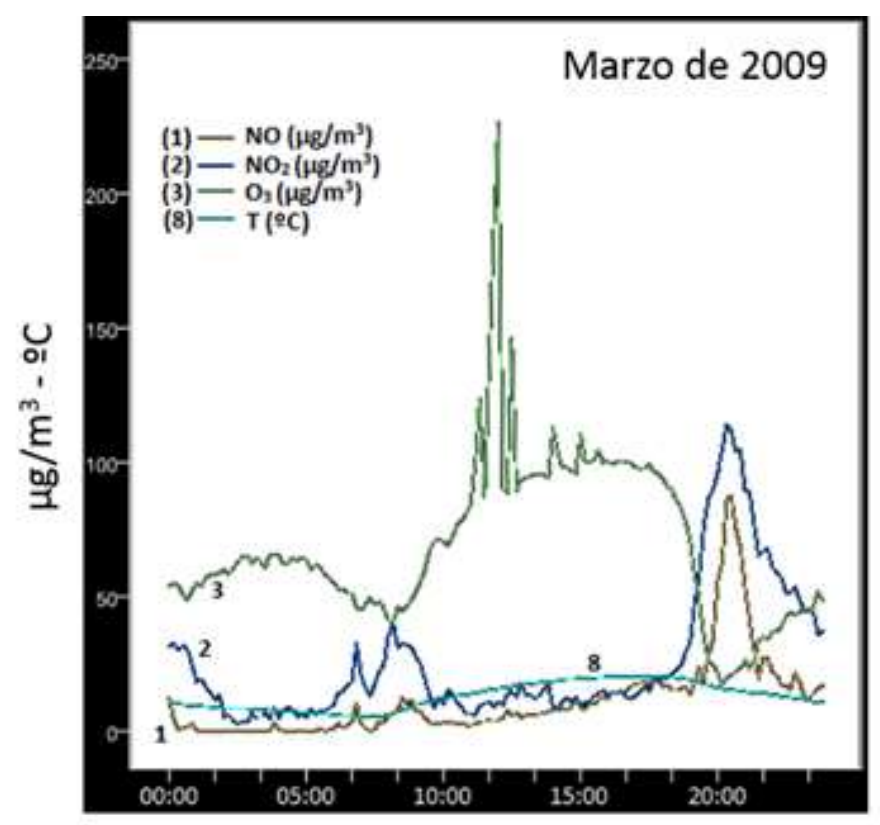

Fig. 6: Gráfica horaria de la temperatura del aire y de los contaminantes indicados

En las horas centrales del día es el ozono (3), el contaminante con mayor concentración, con algunos picos esporádicos, aunque la media horaria no alcance los niveles máximos permitidos por ley. También se observa que los $\mathrm{NO}_{x}(1,2)$, $\mathrm{NO}$ contaminante primario y $\mathrm{NO}_{2}$ secundario, presentan sus mayores concentraciones en las zonas de mínimos para el ozono, confirmando su interrelación. Dado que en la zona 
objeto de estudio se da un clima continental, la radiación solar más intensa corresponderá al mes de mayo (final de primavera) y las de menor intensidad se darán en marzo (final del invierno) y en noviembre (final del otoño). Este hecho deberá tener repercusión en las concentraciones medidas para el ozono y para los óxidos de nitrógeno. Eso es lo que mostramos en las siguientes gráficas. Como se puede observar comparando los datos horarios promediados a los siete días de la semana de los meses de marzo, Fig. 7 y de mayo, Fig.8, la intensidad de la radiación solar (4) es mayor en mayo, y también son más elevadas las concentraciones de ozono, contaminante más dependiente de este factor climático, ya que la disociación fotolítica de otras moléculas además del dióxido de nitrógeno, favorece su formación. En todos los casos analizados las concentraciones están por debajo de los límites que establece el Real Decreto 102/2011. Estos límites de concentraciones permitidas son: $200 \mu \mathrm{g} / \mathrm{m}^{3}$ límite horario para el $\mathrm{NO}_{2}$ (18 superaciones máximas al año) y $120 \mathrm{\mu g} / \mathrm{m}^{3}$ límite horario para el ozono (25 superaciones máximas por año); se puede observar que en el mes de mayo este último contaminante se encuentra cercano al límite establecido. Estos niveles de ozono suelen superarse en los tres meses posteriores.

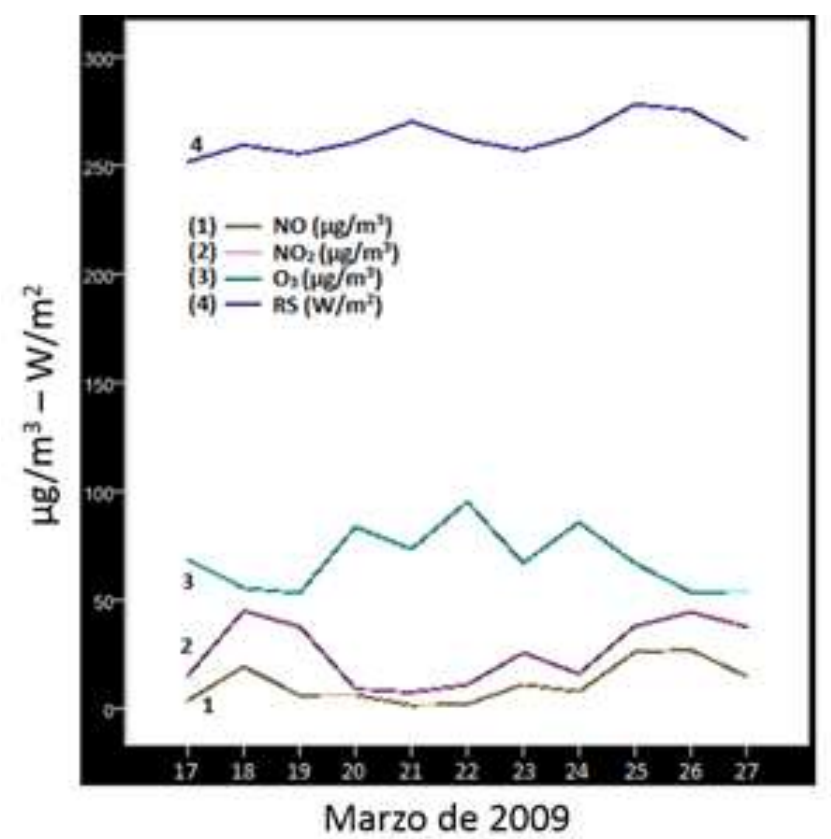

Fig.7: Gráfica semanal promedio de la intensidad solar y de los contaminantes en marzo

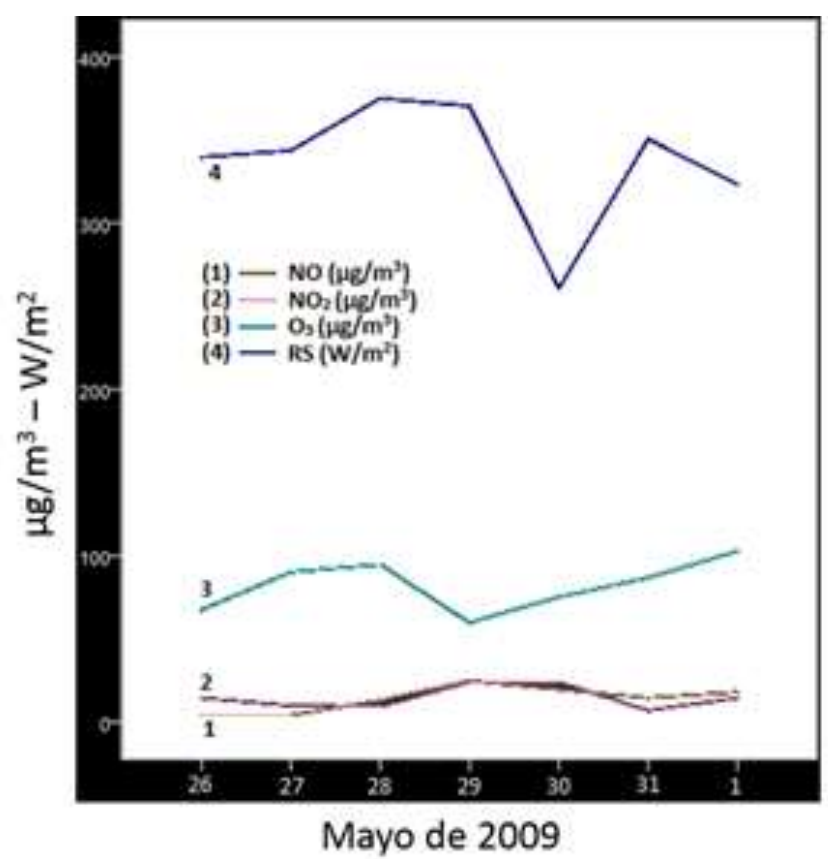

Fig. 8: Gráfica semanal promedio de la intensidad solar y de los contaminantes en mayo

A continuación se hará un análisis más sucinto sobre los gráficos obtenidos por el tratamiento de los datos recogidos para dióxido de azufre y las partículas en suspensión $\mathrm{PM}_{10}$. En la fig. 9 podemos observar la 
variación de la concentración del $\mathrm{SO}_{2}$ a lo largo de una jornada de lunes en los tres periodos mensuales de datos recogidos. Es una constancia en la medida de este contaminante que su mayor concentración se mide a partir de las 17-18h de la tarde, por un proceso acumulativo originado a lo largo de un día sin lluvia, en el que influye la existencia de viento. Hemos constatado que las tardes con viento del norte, que pueden aportar al campus la contaminación de las autopistas que lo rodean, son los días con picos más pronunciados, dentro del hecho de que es el contaminante con menor concentración, por las razones ya indicadas en la introducción. La presencia de lluvia lo elimina de la atmósfera, al reaccionar con las moléculas de agua para formar los ácidos sulfuroso y sulfúrico (lluvia ácida).En la Fig.10 se observa la concentración horaria de las partículas en suspensión $\mathrm{PM}_{10}$, para un miércoles de los tres periodos diferentes analizados.

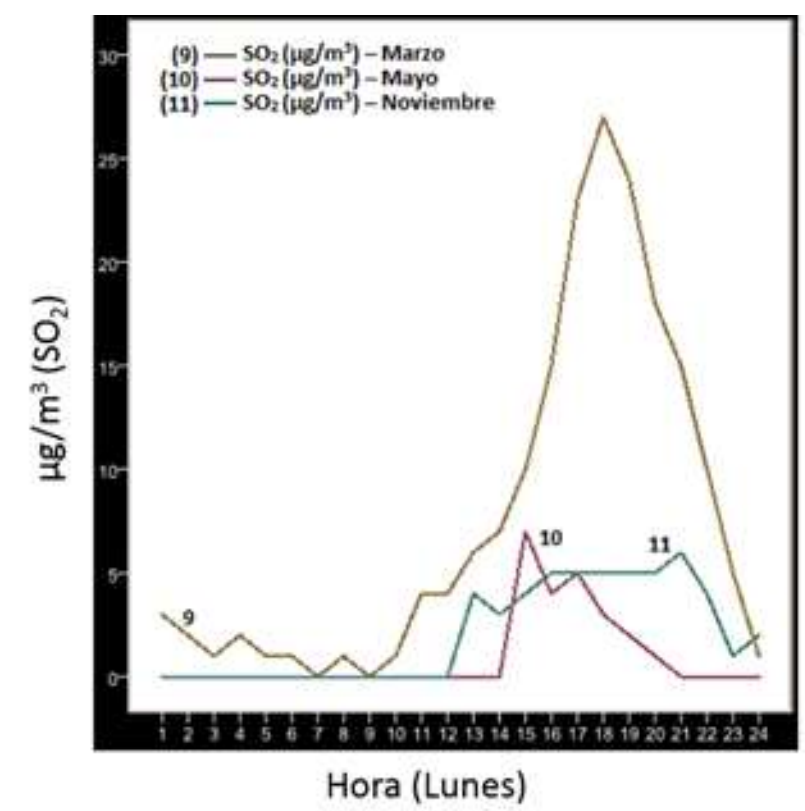

Fig. 9: Gráfico horario del $\mathrm{SO}_{2}$ un lunes de los tres meses analizados

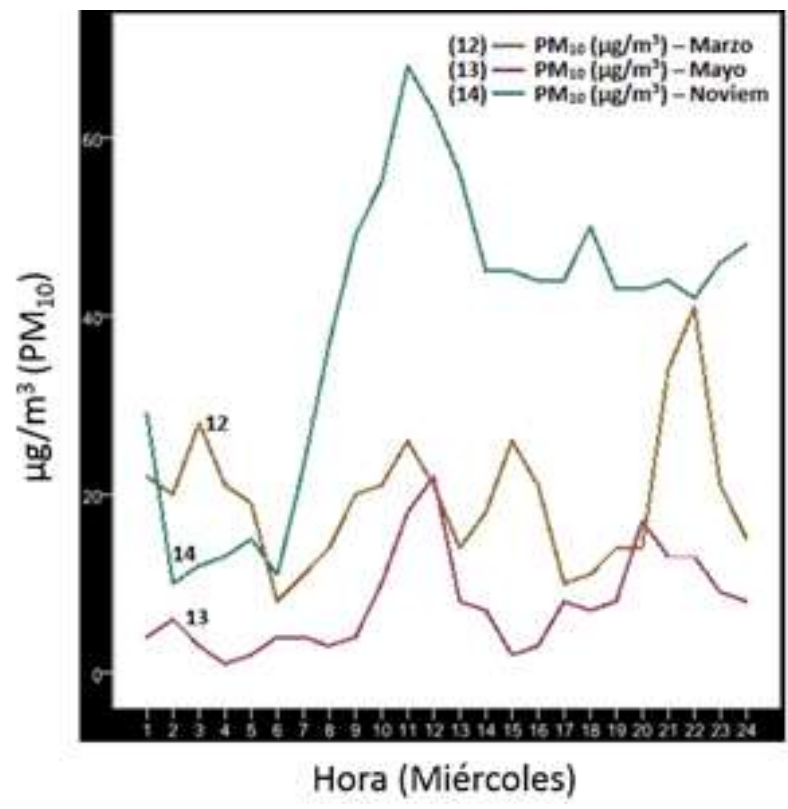

Fig. 10: Gráfico horario de partículas $\mathrm{PM}_{10}$ un miércoles de los tres meses analizados

La máxima concentración se produce en el mes de noviembre, el más frío de los tres periodos elegidos y en el que el uso de transporte privado y el tiempo de encendido de la calefacción es máximo. Le sigue en concentración de este contaminante el mes de marzo y el de menor concentración es el de mayo. Se puede observar que el pico máximo se sitúa entre las 8 y las $11 \mathrm{~h}$ en los tres periodos, coincidiendo con el horario de máxima entrada de vehículos al campus, ver Fig.5. Hay que señalar que este es el único contaminante que supera los límites establecidos en el Real Decreto 102/2011, que es de $50 \mu \mathrm{g} / \mathrm{m}^{3}$ diario. Las superaciones se produjeron en el mes de noviembre y en dos de los días de medición. Uno de ellos está en la Fig. 10. En la Fig. 11 se puede observar la distribución promedio horaria de las partículas PM10 para una 
semana de los tres periodos diferentes analizados. Al igual que en la Fig.10, la mayor concentración de partículas en aire corresponde al mes de noviembre de manera clara y la menor al mes de mayo, si bien los primeros días de la semana para este mes coincidieron con días de vacaciones que explican el mínimo de partículas en aire. De estas observaciones se puede deducir la influencia de la climatología en los usos tanto de vehículos como en el encendido de calefacciones y de ello la repercusión en la mayor concentración de partículas en aire. También influye el factor geográfico, pues el campus se encuentra situado en una ligera hondonada que en días sin viento favorece la acumulación de los contaminantes, por la falta de intercambio de masas de aire.

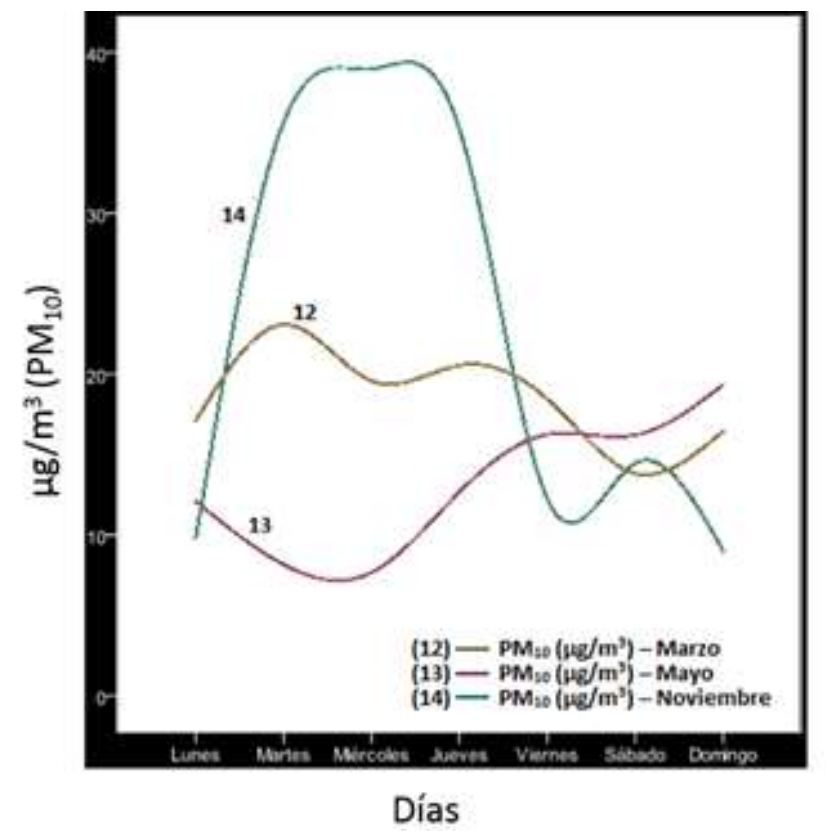

Fig. 11: Gráfico promedio semanal de $\mathrm{PM}_{10}$ de los tres meses analizados

\section{CONCLUSIONES}

La concentración de contaminantes en aire puede ser detectada con precisión a través de los analizadores automáticos, que aportan en intervalos de tiempo real cortos, las concentraciones presentes en la zona elegida para el análisis. Al representar los datos recogidos de los analizadores automáticos para los contaminantes considerados, podemos extraer conclusiones para la zona geográfica seleccionada. Para el caso que se presenta las conclusiones son : a) La contaminación ambiental es baja en el campus de la UAM; b) Las partículas en suspensión $\mathrm{PM}_{10}$ son el único contaminante que sobrepasó el límite establecido por Ley en dos de los días del mes de noviembre de todos los analizados, seguido en concentración próxima a los límites legales por el ozono; c) Las medidas de $\mathrm{NO}_{2}$ y de $\mathrm{O}_{3}$ confirman para todos los periodos la influencia de la radiación solar en el ciclo fotolítico a través del cual se generan o se destruyen según la teoría que lo sustenta. También se observa su correlación con los datos del número de vehículos de acceso al campus; d) Se ha confirmado que el $\mathrm{SO}_{2}$ es el contaminante con menor concentración en el campus.

El análisis de las gráficas muestra cómo los factores climáticos, junto a la hora del día, repercuten en los datos de concentración de las moléculas implicadas en el ciclo fotolítico, así como en la concentración de partículas en suspensión. Todo ello confirma que las medidas directas de los contaminantes en aire, pueden ayudar a la comprensión de su origen, evolución y concentración final, según el espacio físico o temporal en el que se realicen. En el entorno elegido para este análisis, el objetivo de disminuir la concentración de contaminantes debiera llevar a implementar medidas para disminuir el tráfico de vehículos privados en favor del transporte público.

\section{REFERENCIAS}

ABC, Periódico Electrónico SA. El aire contaminado nos endurece las arterias y favorece el riesgo de ictus. Publicado, 6/03/2015 (2015)

AENOR, Asociación Española de Normalización y Certificación. Gestión Ambiental. España (2006)

Atkins P. y Jones L. Principios de Química: Los caminos del descubrimiento. 5a Edición. Ed. Médica Panamericana (2012) 
Atienza J. Recogida y análisis horario de los Contaminantes Atmosféricos $\mathrm{SO}_{2}$ y $\mathrm{PM}_{10}$ entorno al Campus de la UAM y su influencia en la salud. Proyecto fin de carrera. Facultad de Ciencias. UAM. España (2010)

Agencia Europea de Medio Ambiente. El Medio Ambiente en la Unión Europea en el umbral del siglo XXI. Ministerio de Medio Ambiente. España. (2001)

Baird C. Química Ambiental. Ed. Reverte. (2001)

Ballester F., Tenías J., y Pérez S. Efectos de la Contaminación Atmosférica sobre la salud: una introducción. Doi: 10.1590/s113s-57271999000200002, Rev. Esp. de Salud Pública. 121-132 (1999)

Bin Luo et all, Rat Lung Response to $P M_{2.5}$. Exposure under Different cold stresses, Doi:10.3390/ijerph111212927, Int. J. Environ. Res. Public Health, 11(12) 12927-12957 (2014)

Born M. and Wolf E., Principles of Optics. Cambridge University Press (2002)

Figueruelo, J. E. y Marino, M., Química Física del Medio Ambiente y de los procesos Medio Ambientales. Ed. Reverte (2004)

Finlayson-Pitts B. J. and Pitts J. N. Chemistry of the Upper and Lower Atmosphere. Academic Press, San Diego (2000)

Künzli N. and Tager, I. B. Air pollution: from lung to heart. Swiss Med Wkly. 10,135 (47-48) 697-702 (2005)

Künzli, N., Is air pollution on the 20 th century a cause of current asthma hospitalizations? Doi:10.1136/thoraxjnl-2011-200919, Thorax, 67, 2-3 (2012)

Lave L. B. and Seskin E. P. Air Pollution and human health. Sciencie. 723-729 (1970)

Lin-Yu Xu et all, Health Risk Assessment on Inhalable Particulate Matter in Beijing Based on the Thermal Environment, Doi:10.3390/ijrph111212368, Int. J. Environ. Res. Public Health, 11(12) 12368-12388, (2014)

Fernández O. Análisis de la Contaminación Ambiental por óxidos de nitrógeno y ozono en el Campus de Cantoblanco de la UAM. Proyecto fin de carrera. Facultad de Ciencias. UAM. España (2010)

Gass K. et all, Associations between ambient air pollutant mixtures and pediatric asthma emergency department visits in three cities: a classification and regression tree approach, Doi:101186/s12940-0150044-5, Environ. Health, 1456-1470 (2015)

Real Decreto 102/2011. Publicado, 28 de enero de 2011. Boletín Oficial del Estado, España (2011)

Romero Barahona A., Tráfico y transporte en la Universidad Autónoma de Madrid: Hacia una movilidad sostenible. Proyecto fin de carrera. Facultad de Ciencias. UAM. España (2005)

Servicio de Estudios de la Caixa, Anuario Económico de España 2009: Selección de indicadores. La Caixa, España (2009).

SIR 1, (Sistemas de Instalaciones y Redes S. L.). Equipo de Adquisición de datos. Modelo S-4003 (2010).

SIR 2, Dasibi, Modelo 2108. Manual de Operación y mantenimiento. Analizador automático de $\mathrm{NO}_{x}(2001)$.

SIR 3, Dasibi, Modelo 1008RS. Manual de Operación y mantenimiento. Analizador automático de $\mathrm{O}_{3}$ (2001).

SIR 4. Dasibi, Modelo 4108. Manual de Operación y mantenimiento. Analizador automático de $\mathrm{SO}_{2}(2001)$.

Spiro T. G. y Stigliani W. M. Química Medioambiental. Pearson Prentice Hall (2003)

Steinle S. et al. Personal exposure monitoring of $P M_{2.5}$ in indoor and outdoor microenvironments. Dx.doi.org/10.1016/j.scitotenv.2014.12.003, Science of the Total Environment 508, 383-394 (2015)

Tasic, V., et all, Analysis of $\mathrm{SO}_{2}$ Concentration in the Urban Areas near Copper Mining and smelting Complex Bor, Serbia, Doi:103303/CET1442018, Chem. Ingin. Transaction, 42, 103-108 (2014)

Vineis P., Hock, G. and Krzyzanowski, M., Air pollution and risk of lung cancer in a prospective study in Europe, www.ncbi.nlm.nih.gov/pubmed/16463382, Int. J. Cancer, 169-174 (2006)

World Health Organization, Acute effects on health of smog episodes. WHO Regional Publications, European series, (1992) 